\title{
Efficacy of Organically Managed Cropping System in Improvement of Soil Health in Ne Hill Region
}

\author{
N. Khumdemo Ezung ${ }^{1}$, J.K. Choudhary ${ }^{1}$, K. Kurmi ${ }^{1}$, \\ Noyingthung Kikon ${ }^{2 *}$ and Moasunep ${ }^{3}$
}

${ }^{1}$ Department of Agronomy, AAU, Jorhat, Assam-785013, India

${ }^{2}$ ICAR Research Complex for NEH Region, Nagaland Centre, Medziphema, Nagaland-797106, India

${ }^{3}$ Department of Agriculture, GON, Kohima, Nagaland-797001, India

*Corresponding author

K e y w o r d s
$\begin{aligned} & \text { Rice, Maize, Greengram, } \\ & \text { Cropping systems, } \\ & \text { Organic, Nitrogen, } \\ & \text { Phosphorous, } \\ & \text { Vermicompost }\end{aligned}$
Article Info
$\begin{aligned} & \text { Accepted: } \\ & \text { 04 August } 2018 \\ & \text { Available Online: } \\ & \text { 10 September } 2018\end{aligned}$

\section{Introduction}

The agricultural production system in the NE hill region is pre-dominantly rainfed and mono-cropped at subsistence level. Slash and burn agriculture is still practiced in almost all the states on steep slopes with reduced cycle of 2-3 years against 10-15 years in the past. Thus, in the north eastern hill region, crop treatments $\mathrm{N}_{2}$ and $\mathrm{P}_{2}$.
Field experiments were conducted during the kharif seasons of 2015 and 2016 to evaluate the efficacy of the organically managed cropping system in improvement of soil health in NE hill region. Four combinations of two cropping systems (C), viz., rice-greengram (C1) and maize-greengram $\left(\mathrm{C}_{2}\right)$ and two organic $\mathrm{N}$ management treatments $(\mathrm{N})$, viz., $75 \% \mathrm{RD}$ through vermicompost $\left(\mathrm{N}_{1}\right)$ and $100 \% \mathrm{RD}$ through vermicompost (N2) and two organic phosphorus management treatments $(\mathrm{P})$ viz., $75 \% \mathrm{RD}$ through vermicompost $\left(\mathrm{P}_{1}\right)$ and 100 $\% \mathrm{RD}$ through vermicompost $\left(\mathrm{P}_{2}\right)$, which was applied to succeeding crop of greengram after the main kharif crops, were evaluated under the trial in split plot design. Yield and yield attributing characteristics of greengram viz., no. of pods/plant, no. of seeds/pod, test weight, seed yield, stover yield and harvest index (HI) were found to be significantly higher under cropping system $C_{2}$ and organic nutrient management treatments $\mathrm{N}_{2}$ and $\mathrm{P}_{2}$ during both the years. Maximum rice and maize equivalent yield of greengram was also recorded under the cropping system $\mathrm{C}_{2}$ and organic nutrient treatments $\mathrm{N}_{2}$ and $\mathrm{P}_{2}$ during both years. Significant increase in soil organic $\mathrm{C}, \mathrm{pH}$, available $\mathrm{N}, \mathrm{P}_{2} \mathrm{O}_{5}$ and $\mathrm{K}_{2} \mathrm{O}$ both before sowing and after harvest of first (rice and maize) and second (greengram) kharif crops was recorded under the cropping system $\mathrm{C} 2$ and organic nutrient management 
productivity of rice and maize has to be increased from the present level. Rice is also cultivated in Jhum under zero input supply and gives very low yield (1-1-1.5 tha $\left.{ }^{-1}\right)$. The productivity of rice in the state of Tripura (2.3 $\left.\mathrm{t} \mathrm{ha}^{-1}\right)$ and Manipur $\left(2.5 \mathrm{tha}^{-1}\right)$ are higher than the national average, whereas all other states have lower productivity compared to national average (Das et al., 2011). In case of maize, the state of Manipur (2.2 $\left.\mathrm{t} \mathrm{ha}^{-1}\right)$, Mizoram (1.8 $\left.\mathrm{t} \mathrm{ha}^{-1}\right)$ and Nagaland (2 $\left.\mathrm{t} \mathrm{ha}^{-1}\right)$, has comparatively better productivity than national average (Das et al., 2011). Simply by adopting low cost agro-techniques like improved variety, proper time of sowing, intercultural practices, effective recycling of resources etc, yield can be increased significantly. In the present scenario of degradation of natural resources, the value of pulses is far more important. Pulses are nutritious food, feed and forage and is an integral component of subsistence cropping systems.The beneficial effect of pulse crops in improving soil health and sustaining productivity has long been realized. Due to qualitative changes in physical, chemical and biological properties, on account of biological nitrogen fixation, addition of considerable amount of organic matter through root biomass and leaf fall, deep root systems, mobilization of nutrients, protection of soil against erosion and improving microbial biomass, soil stay productive and alive. It is, therefore, imperative that grain legumes are given a preference in cropping systems of both irrigated and dryland areas.Farming in the north-east hill region is regarded as organic by default as the application of fertilizers and pesticides is meagre in these parts compared to the other parts of the country. However, with increasing population and reducing production and productivity of traditional systems of crop production in this region, there is need to increase the cropping intensity and convert subsistence agriculture into a sustainable form like organic agriculture.
Organic agriculture is one among the broadspectrum production methods that are supportive of the environment (Ramesh et al., 2010). Hence, the present investigation was conducted to study the efficacy of organically managed cropping system in improvement of soil health in NE hill region

\section{Materials and Methods}

The present investigation was carried out during the kharif seasons of 2015 and 2016 at the experimental farm of ICAR, Nagaland Centre, Medziphema. The climatic condition of the experimental area is sub-tropical humid with annual average rainfall of $1500 \mathrm{~mm}$ to $2000 \mathrm{~mm}$ which is mainly received from April to October the remaining months being generally dry. The mean summer temperature ranges between $19^{\circ} \mathrm{C}$ to $35^{\circ} \mathrm{C}$, while in winter it rarely goes below $5^{\circ} \mathrm{C}$. The soil of the experimental field was sandy loam in texture with $\mathrm{pH} 4.84$, organic carbon $(0.47 \%), \mathrm{N}$ (147.39 $\left.\mathrm{kg} \mathrm{ha}^{-1}\right), \mathrm{P}_{2} \mathrm{O}_{5}\left(19.04 \mathrm{~kg} \mathrm{ha}^{-1}\right)$ and $\mathrm{K}_{2} \mathrm{O}$ $\left(170.02 \mathrm{~kg} \mathrm{ha}^{-1}\right)$. The experiment was laid out in split plot design with three replications. The main treatment included 4 combinations of two cropping systems $(\mathrm{C}), v i z$. , rice-greengram $\left(\mathrm{C}_{1}\right)$ and maize-greengram $\left(\mathrm{C}_{2}\right)$ and two organic $\mathrm{N}$ management treatments $(\mathrm{N}), v i z ., 75$ $\%$ RD through vermicompost $\left(\mathrm{N}_{1}\right)$ and $100 \%$ RD through vermicompost $\left(\mathrm{N}_{2}\right)$ whereas sub plot treatments included two organic phosphorus management treatments (P) viz., $75 \%$ RD through vermicompost $\left(\mathrm{P}_{1}\right)$ and 100 $\%$ RD through vermicompost $\left(\mathrm{P}_{2}\right)$ which was applied to succeeding crop of greengram after the main kharif crops. Combinations of main plot treatments are as follows, C1N1, C1N2, $\mathrm{C} 2 \mathrm{~N} 1$ and $\mathrm{C} 2 \mathrm{~N} 2$. Upland rice variety Inglonkiri, composite maize variety ' $\mathrm{RCM}-76$ ' and green gram variety 'Pratap (SG-1)' were used for the present investigation. For kharif rice and maize the experimental plot was ploughed thoroughly with tractor drawn disc plough and disc harrow followed by laddering 
to obtain fine tilth and a levelled bed suitable whereas, for the following kharif greengram crop, the individual plots were hoed immediatedly after the harvest of the kharif crops and each individual plots, which were considered as main plots, were split into two sub-plots by constructing ridges $20 \mathrm{~cm}$ high and $30 \mathrm{~cm}$ wide. For organic $\mathrm{N}$ and $\mathrm{P}$ management through vermicompost the quantities of vermicompost required for organic $\mathrm{N}$ and $\mathrm{P}$ management were calculated based on based on following recommended nutrient doses viz., rice - $40 \mathrm{~kg} \mathrm{ha}^{-1}$, maize- 60 $\mathrm{kg} \mathrm{ha}{ }^{-1}$ and greengram- $35 \mathrm{~kg} \mathrm{ha}^{-1}$. Yield attributes of green gram viz. number of pods/plant, seeds/pod, test weight, seed yield, stover yield and harvest index and rice and maize equivalent yields of greengram were recorded to access the response of the crop to the different management practices. Soil nutrient status viz., soil organic $\mathrm{C}(\%), \mathrm{pH}$, available $\mathrm{N}, \mathrm{P}_{2} \mathrm{O}_{5}$ and $\mathrm{K}_{2} \mathrm{O}$ were also recorded before and after harvest of each crop to evaluate the efficacy of the different treatments on soil health.

\section{Results and Discussion}

Yield and yield attributing characters of greengram

The effect of the main plot factors viz., cropping systems (C) and organic $\mathrm{N}$ management $(\mathrm{N})$ on the yield attributing characteristics of greengram were found to be significant (Table 1 and 2). Significantly higher number of pods/plant, seeds/pod, test weight, seed yield, stover yield and harvest index $(\mathrm{HI})$ of green gram were recorded under maize-greengram system $\left(\mathrm{C}_{2}\right)$ as compared to rice-greengram system $\left(\mathrm{C}_{1}\right)$. Application of $100 \% \mathrm{~N}$ through vermicompost $\left(\mathrm{N}_{2}\right)$ was also found to record significantly higher yield and yield attributes of greengram during both the years compared to application of $75 \% \mathrm{~N}$ as vermicompost $\left(\mathrm{N}_{1}\right)$. The effect of sub plot factors viz., organic phosphorous management (P) was also found to record significant variations in yield and yield attributes of green gram (Table 1). The data revealed that application of $100 \% \mathrm{P}$ through vermicompost $\left(\mathrm{P}_{2}\right)$ in greengram resulted in significantly more number of pods/plant, number of seeds/pod, test weight, seed yield, stover yield and $\mathrm{HI}$ as compared to the treatment $\mathrm{P}_{1}(75 \%$ $\mathrm{P}$ through vermicompost) during both years.

\section{Rice equivalent yield of greengram}

Data presented in Table 3 shows the rice equivalent yield $\left(\mathrm{q} \mathrm{ha}{ }^{-1}\right)$ of greengram as affected by cropping systems, organic $\mathrm{N}$ management and organic phosphorus management.

The main plot factors significantly influenced the rice equivalent yield of greengram during both the years. It was recorded that significantly higher rice equivalent yield of greengram was obtained from maizegreengram system $\left(\mathrm{C}_{2}\right)$ as compared with ricegreengram system $\left(\mathrm{C}_{1}\right)$. Application of $100 \%$ $\mathrm{N}$ through vermicompost $\left(\mathrm{N}_{2}\right)$ was found to record significantly higher rice equivalent yield of greengram during both the years compared to application of $75 \% \mathrm{~N}$ as vermicompost $\left(\mathrm{N}_{1}\right)$. Application of organic phosphorus in greengram also showed significant influence on the rice equivalent yield of greengram during both the years. It was found that the application of $100 \% \mathrm{P}$ through vermicompost $\left(\mathrm{P}_{2}\right)$ in greengram resulted in higher rice equivalent yield of greengram during both the years compared to application of $75 \% \mathrm{P}$ through vermicompost $\left(\mathrm{P}_{1}\right)$.

\section{Maize equivalent yield of greengram}

The effect of cropping systems, organic $\mathrm{N}$ management and organic phosphorus management on maize equivalent yield ( $q$ 
$\mathrm{ha}^{-1}$ ) of greengram are presented in Table 3. The main plot factors significantly influenced the maize equivalent yield of greengram during both the years. It was recorded that significantly higher maize equivalent yield of greengram was obtained from maizegreengram system $\left(\mathrm{C}_{2}\right)$ as compared with ricegreengram system $\left(\mathrm{C}_{1}\right)$.

It was also revealed that the application of 100 $\% \mathrm{~N}$ through vermicompost $\left(\mathrm{N}_{2}\right)$ recorded significantly higher maize equivalent yield of greengram compared to the application $75 \%$ $\mathrm{N}$ through vermicompost $\left(\mathrm{N}_{1}\right)$. Application of organic phosphorus in greengram showed significant influence on the maize equivalent yield of greengram during both the years. It was found that the application of $100 \% \mathrm{P}$ through vermicompost $\left(\mathrm{P}_{2}\right)$ in greengram resulted in higher maize equivalent yield compared to the application of $75 \% \mathrm{P}$ through vermicompost $\left(\mathrm{P}_{1}\right)$.

\section{Soil nutrient status before sowing and after harvest of first $k$ harif crop rice and maize}

Significant variations were observed with respect to soil organic $\mathrm{C}, \mathrm{pH}$, available $\mathrm{N}$, $\mathrm{P}_{2} \mathrm{O}_{5}$ and $\mathrm{K}_{2} \mathrm{O}$ recorded before and after harvest of rice and maize (Table 4 and 5). Cropping system was found to record significant effect on the soil organic $\mathrm{C}$ and available $\mathrm{N}, \mathrm{P}_{2} \mathrm{O}_{5}$ and $\mathrm{K}_{2} \mathrm{O}$ in soil after harvest of the first kharif crops (rice and maize).

Significantly higher soil organic $\mathrm{C}$ and available $\mathrm{N}, \mathrm{P}_{2} \mathrm{O}_{5}$ and $\mathrm{K}_{2} \mathrm{O}$ was recorded with $\mathrm{C}_{2}$ (maize-greengram) during 2016 however, it was found to be at par with $\mathrm{C}_{1}$ (ricegreengram) during 2015 in case of available $\mathrm{N}$ and $\mathrm{K}_{2} \mathrm{O}$. During the year 2016, before sowing of first kharif crop all soil parameters viz., soil organic $\mathrm{C}, \mathrm{pH}$ and available $\mathrm{N}$ and $\mathrm{P}_{2} \mathrm{O}_{5}$ showed significant differences except for available $\mathrm{K}_{2} \mathrm{O}$ which was found to be at par for both $\mathrm{C}_{1}$ and $\mathrm{C}_{2}$. Organic nitrogen management $(\mathrm{N})$ was also found to record significant variations with respect to soil organic $\mathrm{C}$ and available $\mathrm{N}$ and $\mathrm{P}_{2} \mathrm{O}_{5}$ both before sowing and at harvest of first kharif (rice and maize) during 2016. It was observed that significantly higher soil organic $\mathrm{C}$, available $\mathrm{N}$ and $\mathrm{P}_{2} \mathrm{O}_{5}$ in soil were recorded with application of $100 \% \quad \mathrm{~N}$ through vermicompost $\left(\mathrm{N}_{2}\right)$ compared with $\mathrm{N}_{1}(75 \%$ $\mathrm{N}$ through vermicompost) however, during 2015 after harvest and 2016 before sowing both $\mathrm{N}_{1}$ and $\mathrm{N}_{2}$ were found to be at par with respect to soil available $\mathrm{K}_{2} \mathrm{O}$.

\section{Soil nutrient status before sowing and after harvest of second $k$ harif crop greengram}

Cropping systems, organic nitrogen management and organic phosphorous management were found to record significant differences with respect to the different soil parameters both before sowing (after harvest of rice and maize) and after harvest of second kharif crop greengram during both years (Table 6 and 7). During both years, it was observed that soil organic $\mathrm{C}, \mathrm{pH}$, available $\mathrm{N}$, $\mathrm{P}_{2} \mathrm{O}_{5}$ and $\mathrm{K}_{2} \mathrm{O}$ of soil both before sowing and after harvest of greengram was significantly higher in case of maize-greengram system $\left(\mathrm{C}_{2}\right)$ as compared to rice-greengram $\operatorname{system}\left(\mathrm{C}_{1}\right)$.

Organic nitrogen management $\mathrm{N}_{2}(100 \% \mathrm{~N}$ through vermicompost) also showed significantly higher soil organic $\mathrm{C}, \mathrm{pH}$, available $\mathrm{N}, \mathrm{P}_{2} \mathrm{O}_{5}$ and $\mathrm{K}_{2} \mathrm{O}$ before sowing and after harvest of greengram as compared to $\mathrm{N}_{1}$ (75\% $\mathrm{N}$ through vermicompost) during both the years. With regard to organic phosphorous management, during both years it was also observed that significantly higher organic $\mathrm{C}$, $\mathrm{pH}$, available $\mathrm{N}, \mathrm{P}_{2} \mathrm{O}_{5}$ and $\mathrm{K}_{2} \mathrm{O}$ was recorded from the application of $100 \% \mathrm{P}$ through vermicompost $\left(\mathrm{P}_{2}\right)$ as compared to application of $75 \% \mathrm{P}$ through vermicompost $\left(\mathrm{P}_{1}\right)$ both before sowing and after harvest of greengram. 
Table.1 Effect of cropping system, organic $\mathrm{N}$ and weed management on yield parameters of greengram

\begin{tabular}{|c|c|c|c|c|c|c|}
\hline \multirow[b]{2}{*}{ Treatment } & \multicolumn{2}{|c|}{ Number of pods/plant } & \multicolumn{2}{|c|}{ Number of seeds/pod } & \multicolumn{2}{|c|}{ Test weight (g) } \\
\hline & 2015 & 2016 & 2015 & 2016 & 2015 & 2016 \\
\hline \multicolumn{7}{|l|}{ Cropping system (C) } \\
\hline $\mathrm{C}_{1}$-Rice-greengram & 28.701 & 23.032 & 9.848 & 7.552 & 34.392 & 31.372 \\
\hline $\mathrm{C}_{2}$-Maize-greengram & 31.331 & 26.784 & 10.162 & 8.326 & 35.767 & 32.762 \\
\hline \multicolumn{7}{|c|}{ Organic $N$ management in $1^{s t}$ kharif crop $(N)$} \\
\hline $\mathbf{N}_{1-75} \mathbf{N}$ as vermicompost & 28.749 & 23.888 & 9.787 & 7.711 & 34.216 & 31.199 \\
\hline $\mathbf{N}_{2}-100 \% \mathrm{~N}$ as vermicompost & 31.283 & 25.928 & 10.224 & 8.137 & 35.943 & 32.935 \\
\hline $\operatorname{SEm}( \pm)$ & 0.763 & 0.602 & 0.144 & 0.139 & 0.512 & 0.516 \\
\hline $\mathrm{CD}(\mathrm{P}=0.05)$ & 1.634 & 1.288 & 0.309 & 0.297 & 1.095 & 1.105 \\
\hline \multicolumn{7}{|c|}{ Organic $P$ management in $2^{\text {nd }}$ kharif crop $(P)$} \\
\hline$P_{1-} \mathbf{7 5} \% \mathbf{P}$ as vermicompost & 27.723 & 22.890 & 9.677 & 7.611 & 33.851 & 30.838 \\
\hline$P_{2^{-}} 100 \% P$ as vermicompost & 32.309 & 26.926 & 10.334 & 8.237 & 36.309 & 33.295 \\
\hline $\operatorname{SEm}( \pm)$ & 0.412 & 0.526 & 0.197 & 0.127 & 0.395 & 0.439 \\
\hline $\mathrm{CD}(\mathrm{P}=0.05)$ & 0.874 & 1.127 & 0.417 & 0.272 & 0.838 & 0.939 \\
\hline Interactions & NS & NS & NS & NS & NS & NS \\
\hline \multirow[t]{2}{*}{ CV $(\%)$} & 8.811 & 8.370 & 5.001 & 6.074 & 5.051 & 5.576 \\
\hline & 4.757 & 7.322 & 6.814 & 5.565 & 3.903 & 4.739 \\
\hline
\end{tabular}


Table.2 Effect of cropping system, organic $\mathrm{N}$ and $\mathrm{P}$ management on yield parameters of greengram

\begin{tabular}{|c|c|c|c|c|c|c|}
\hline \multirow[t]{2}{*}{ Treatment } & \multicolumn{2}{|c|}{ Seed yield $\left(\mathbf{q} \mathbf{h a}^{-1}\right)$} & \multicolumn{2}{|c|}{ Stover yield (q ha $\left.{ }^{-1}\right)$} & \multicolumn{2}{|c|}{ Harvest Index (\%) } \\
\hline & 2015 & 2016 & 2015 & 2016 & 2015 & 2016 \\
\hline \multicolumn{7}{|l|}{ Cropping system $(C)$} \\
\hline $\mathrm{C}_{1}$-Rice-greengram & 9.218 & 6.782 & 19.307 & 17.010 & 29.716 & 27.730 \\
\hline $\mathrm{C}_{2}$-Maize-greengram & 9.542 & 7.497 & 22.262 & 19.376 & 32.282 & 28.648 \\
\hline \multicolumn{7}{|c|}{ Organic $N$ management in $I^{\text {st }}$ kharif crop $(N)$} \\
\hline $\mathrm{N}_{1-} \mathbf{7 5} \% \mathrm{~N}$ as vermicompost & 9.164 & 6.871 & 20.383 & 17.770 & 30.294 & 27.786 \\
\hline $\mathrm{N}_{2}-\mathbf{1 0 0 \%} \mathrm{N}$ as vermicompost & 9.595 & 7.408 & 21.186 & 18.616 & 31.704 & 28.591 \\
\hline $\operatorname{SEm}( \pm)$ & 0.124 & 0.144 & 0.379 & 0.284 & 0.422 & 0.365 \\
\hline $\mathrm{CD}(\mathrm{P}=\mathbf{0 . 0 5})$ & 0.265 & 0.307 & 0.810 & 0.609 & 0.903 & 0.782 \\
\hline \multicolumn{7}{|c|}{ Organic $P$ management in $2^{\text {nd }}$ kharif crop $(P)$} \\
\hline $\mathbf{P}_{1-75} \mathbf{P}$ as vermicompost & 8.984 & 6.719 & 19.922 & 17.463 & 29.626 & 27.291 \\
\hline$P_{2^{-}} 100 \% P$ as vermicompost & 9.775 & 7.560 & 21.648 & 18.923 & 32.372 & 29.087 \\
\hline $\operatorname{SEm}( \pm)$ & 0.150 & 0.121 & 0.503 & 0.390 & 0.483 & 0.383 \\
\hline $\mathrm{CD}(\mathrm{P}=\mathbf{0 . 0 5})$ & 0.317 & 0.260 & 1.067 & 0.835 & 1.023 & 0.820 \\
\hline Interactions & NS & NS & NS & NS & NS & NS \\
\hline \multirow[t]{2}{*}{$\mathrm{CV}(\%)$} & 4.581 & 6.967 & 6.304 & 5.416 & 4.718 & 4.488 \\
\hline & 5.523 & 5.890 & 8.383 & 7.429 & 5.393 & 4.709 \\
\hline
\end{tabular}

NS- Not significant 
Table.3 Effect of cropping system, organic $\mathrm{N}$ and $\mathrm{P}$ management on rice and maize equivalent yield of greengram

\begin{tabular}{|c|c|c|c|c|}
\hline \multirow[t]{2}{*}{ Treatment } & \multicolumn{2}{|c|}{ Rice equivalent yield (q ha $\left.{ }^{-1}\right)$} & \multicolumn{2}{|c|}{ Maize equivalent yield $\left(\mathbf{q} \mathbf{h a}^{-1}\right)$} \\
\hline & 2015 & 2016 & 2015 & 2016 \\
\hline \multicolumn{5}{|l|}{ Cropping system $(C)$} \\
\hline $\mathrm{C}_{1}$-Rice-greengram & 30.725 & 22.607 & 21.104 & 15.651 \\
\hline $\mathrm{C}_{2}$-Maize-greengram & 31.805 & 24.809 & 21.903 & 17.301 \\
\hline \multicolumn{5}{|c|}{ Organic $N$ management in $1^{\text {st }}$ kharif crop $(N)$} \\
\hline $\mathrm{N}_{1-} \mathbf{7 5} \% \mathrm{~N}$ as vermicompost & 30.547 & 22.722 & 21.032 & 15.856 \\
\hline $\mathbf{N}_{\mathbf{2}}-\mathbf{1 0 0 \%} \mathrm{N}$ as vermicompost & 31.983 & 24.694 & 21.975 & 17.096 \\
\hline $\operatorname{SEm}( \pm)$ & 0.413 & 0.488 & 0.204 & 0.331 \\
\hline $\mathrm{CD}(\mathrm{P}=\mathbf{0 . 0 5})$ & 0.885 & 1.044 & 0.437 & 0.709 \\
\hline \multicolumn{5}{|c|}{ Organic $P$ management in $2^{\text {nd }}$ kharif crop $(P)$} \\
\hline$P_{1-75 \%} P$ as vermicompost & 29.947 & 22.287 & 20.594 & 15.506 \\
\hline$P_{2^{-}} 100 \% P$ as vermicompost & 32.583 & 25.129 & 22.413 & 17.466 \\
\hline $\operatorname{SEm}( \pm)$ & 0.499 & 0.401 & 0.379 & 0.280 \\
\hline $\mathrm{CD}(\mathrm{P}=0.05)$ & 1.067 & 0.859 & 0.811 & 0.599 \\
\hline Interactions & NS & NS & NS & NS \\
\hline \multirow[t]{2}{*}{ CV $(\%)$} & 4.581 & 7.126 & 3.292 & 6.967 \\
\hline & 5.523 & 5.864 & 6.106 & 5.890 \\
\hline
\end{tabular}

NS- Not significant 
Table.4 Soil nutrient status before sowing of first kharif crops (rice and maize) during 2016

\begin{tabular}{|c|c|c|c|c|c|}
\hline Treatment & $\mathrm{OC}(\%)$ & pH & $\begin{array}{l}\text { Available } \mathbf{N} \\
\left(\mathrm{kg} \mathrm{ha}^{-1}\right)\end{array}$ & $\begin{array}{c}\text { Available } \\
\mathrm{P}_{2} \mathrm{O}_{5}\left(\mathrm{~kg} \mathrm{ha}^{-1}\right)\end{array}$ & $\begin{array}{c}\text { Available } \mathrm{K}_{2} \mathbf{O} \\
\left(\mathrm{kg} \mathrm{ha}^{-1}\right)\end{array}$ \\
\hline \multicolumn{6}{|l|}{ Cropping system $(C)$} \\
\hline $\mathrm{C}_{1}$-Rice-Greengram & 0.604 & 5.503 & 259.318 & 31.355 & 147.702 \\
\hline $\mathrm{C}_{2}$-Maize-Greengram & 0.626 & 5.577 & 263.384 & 33.799 & 150.578 \\
\hline \multicolumn{6}{|c|}{ Organic $N$ management during $I^{s t}$ kharif crop (N) } \\
\hline $\mathrm{N}_{1}-\mathbf{7 5} \% \mathrm{~N}$ as vermicompost & 0.603 & 5.496 & 259.013 & 31.361 & 147.727 \\
\hline $\mathbf{N}_{2^{-}} 100 \% \mathrm{~N}$ as vermicompost & 0.625 & 5.581 & 263.689 & 33.801 & 150.553 \\
\hline $\operatorname{SEm}( \pm)$ & 0.004 & 0.019 & 0.089 & 0.444 & 0.921 \\
\hline $\mathrm{CD}(\mathrm{P}=\mathbf{0 . 0 5})$ & 0.020 & 0.084 & 4.010 & 1.988 & 4.144 \\
\hline Interaction & NS & NS & NS & NS & NS \\
\hline $\mathrm{CV}(\%)$ & 2.461 & 1.167 & 1.182 & 4.723 & 2.139 \\
\hline
\end{tabular}

NS- Not significant

Table.5 Soil nutrient status after harvest of $1^{\text {st }}$ kharif crops (rice and maize)

\begin{tabular}{|c|c|c|c|c|c|c|c|c|c|c|}
\hline Treatment & \multicolumn{2}{|c|}{$\mathrm{OC}(\%)$} & \multicolumn{2}{|c|}{$\mathrm{pH}$} & \multicolumn{2}{|c|}{$\begin{array}{l}\text { Available } \mathbf{N} \\
\left(\mathrm{kg} \mathrm{ha}^{-1}\right)\end{array}$} & \multicolumn{2}{|c|}{$\begin{array}{l}\text { Available } \mathrm{P}_{2} \mathrm{O}_{5} \\
\left(\mathrm{~kg} \mathrm{ha}^{-1}\right)\end{array}$} & \multicolumn{2}{|c|}{$\begin{array}{l}\text { Available } \mathrm{K}_{2} \mathrm{O} \\
\left(\mathrm{kg} \mathrm{ha}^{-1}\right)\end{array}$} \\
\hline \multicolumn{11}{|l|}{ Cropping system $(C)$} \\
\hline $\mathrm{C}_{2}$-Maize-Greengram & 0.539 & 0.640 & 5.298 & 5.619 & 258.539 & 270.578 & 23.923 & 35.799 & 133.878 & 162.361 \\
\hline \multicolumn{11}{|c|}{$\begin{array}{l}\text { Organic } N \text { management during } I^{\text {st }} \\
\text { kharif } \operatorname{crop}(N)\end{array}$} \\
\hline $\operatorname{SEm}( \pm)$ & 0.002 & 0.004 & 0.025 & 0.017 & 1.592 & 0.841 & 0.326 & 0.458 & 1.287 & 0.825 \\
\hline $\mathrm{CD}(\mathrm{P}=0.05)$ & 0.010 & 0.018 & 0.111 & 0.078 & 7.165 & 3.785 & 1.465 & 2.060 & 5.790 & 3.713 \\
\hline Interaction & NS & NS & NS & NS & NS & NS & NS & NS & NS & NS \\
\hline $\mathrm{CV}(\%)$ & 1.481 & 2.154 & 1.633 & 1.075 & 2.154 & 1.086 & 4.901 & 4.586 & 3.381 & 1.920 \\
\hline
\end{tabular}


Table.6 Soil nutrient status before sowing of second kharif crop greengram

\begin{tabular}{|c|c|c|c|c|c|c|c|c|c|c|}
\hline \multirow[t]{2}{*}{ Treatment } & \multicolumn{2}{|c|}{$\mathrm{OC}(\%)$} & \multicolumn{2}{|c|}{ pH } & \multicolumn{2}{|c|}{$\begin{array}{c}\text { Available } \mathbf{N} \\
\left(\mathrm{kg} \mathrm{ha}^{-1}\right)\end{array}$} & \multicolumn{2}{|c|}{$\begin{array}{c}\text { Available } \mathrm{P}_{2} \mathrm{O}_{5} \\
\left(\mathrm{~kg} \mathrm{ha}^{-1}\right)\end{array}$} & \multicolumn{2}{|c|}{$\begin{array}{l}\text { Available } \mathrm{K}_{2} \mathrm{O} \\
\left(\mathrm{kg} \mathrm{ha}^{-1}\right)\end{array}$} \\
\hline & 2015 & 2016 & 2015 & 2016 & 2015 & 2016 & 2015 & 2016 & 2015 & 2016 \\
\hline \multicolumn{11}{|l|}{ Cropping system $(C)$} \\
\hline $\mathrm{C}_{1}$-Rice-greengram & 0.540 & 0.609 & 5.274 & 5.510 & 250.935 & 256.535 & 23.822 & 32.427 & 129.517 & 163.933 \\
\hline $\mathrm{C}_{2}$-Maize-greengram & 0.556 & 0.633 & 5.300 & 5.590 & 258.187 & 263.683 & 25.048 & 33.512 & 138.464 & 172.756 \\
\hline \multicolumn{11}{|c|}{$\begin{array}{l}\text { Organic } N \text { management in } \\
I^{\text {st }} \text { kharif } \operatorname{crop}(N)\end{array}$} \\
\hline $\begin{array}{l}\mathrm{N}_{1}-75 \% \mathrm{~N} \text { as } \\
\text { vermicompost } \\
\end{array}$ & 0.539 & 0.614 & 5.269 & 5.522 & 252.452 & 258.879 & 23.997 & 32.457 & 132.746 & 167.246 \\
\hline $\begin{array}{l}\mathrm{N}_{2}-100 \% \mathrm{~N} \text { as } \\
\text { vermicompost } \\
\end{array}$ & 0.556 & 0.628 & 5.305 & 5.568 & 256.670 & 261.340 & 24.894 & 33.483 & 135.235 & 169.443 \\
\hline $\operatorname{SEm}( \pm)$ & 0.004 & 0.004 & 0.008 & 0.013 & 1.302 & 0.783 & 0.232 & 0.233 & 1.139 & 1.014 \\
\hline $\mathrm{CD}(\mathrm{P}=0.05)$ & 0.012 & 0.009 & 0.025 & 0.039 & 2.785 & 1.677 & 0.496 & 0.498 & 2.437 & 2.169 \\
\hline \multicolumn{11}{|c|}{$\begin{array}{l}\text { Organic P management in } \\
2^{\text {nd }} \text { kharif crop }(P)\end{array}$} \\
\hline $\begin{array}{l}P_{1-75 \%} P \text { as } \\
\text { vermicompost }\end{array}$ & 0.538 & 0.609 & 5.248 & 5.518 & 252.259 & 257.565 & 23.461 & 31.919 & 131.718 & 165.718 \\
\hline $\begin{array}{l}P_{2^{-}} 100 \% \mathrm{P} \text { as } \\
\text { vermicompost }\end{array}$ & 0.557 & 0.633 & 5.326 & 5.583 & 256.863 & 262.653 & 25.410 & 34.020 & 136.263 & 170.971 \\
\hline $\operatorname{SEm}( \pm)$ & 0.004 & 0.004 & 0.011 & 0.013 & 1.564 & 0.856 & 0.200 & 0.205 & 0.869 & 0.662 \\
\hline $\mathrm{CD}(\mathrm{P}=0.05)$ & 0.011 & 0.009 & 0.034 & 0.039 & 3.316 & 1.832 & 0.428 & 0.438 & 1.841 & 1.416 \\
\hline Interactions & NS & NS & NS & NS & NS & NS & NS & NS & NS & NS \\
\hline \multirow[t]{2}{*}{$\mathrm{CV}(\%)$} & 3.651 & 2.413 & 0.766 & 1.144 & 1.771 & 1.043 & 3.288 & 2.446 & 2.944 & 2.086 \\
\hline & 3.341 & 2.271 & 1.054 & 1.148 & 2.128 & 1.140 & 2.833 & 2.150 & 2.245 & 1.361 \\
\hline
\end{tabular}


Table.7 Soil nutrient status after harvesting of second kharif crop greengram

\begin{tabular}{|c|c|c|c|c|c|c|c|c|c|c|}
\hline \multirow[t]{2}{*}{ Treatment } & \multicolumn{2}{|c|}{$\mathrm{OC}(\%)$} & \multicolumn{2}{|c|}{ pH } & \multicolumn{2}{|c|}{$\begin{array}{l}\text { A vailable } \mathbf{N} \\
\left(\mathrm{kg} \mathrm{ha}^{-1}\right)\end{array}$} & \multicolumn{2}{|c|}{$\begin{array}{l}\text { Available } \mathrm{P}_{2} \mathrm{O}_{5} \\
\left(\mathrm{~kg} \mathrm{ha}^{-1}\right)\end{array}$} & \multicolumn{2}{|c|}{$\begin{array}{l}\text { Available } \mathrm{K}_{2} \mathbf{O} \\
\left(\mathrm{kg} \mathrm{ha}^{-1}\right)\end{array}$} \\
\hline & 2015 & 2016 & 2015 & 2016 & 2015 & 2016 & 2015 & 2016 & 2015 & 2016 \\
\hline \multicolumn{11}{|l|}{ Cropping system (C) } \\
\hline $\mathrm{C}_{1}$-Rice-greengram & 0.578 & 0.716 & 5.380 & 5.760 & 258.698 & 265.433 & 33.704 & 38.150 & 157.860 & 169.855 \\
\hline $\mathrm{C}_{2}$-Maize-greengram & 0.595 & 0.736 & 5.429 & 5.840 & 266.127 & 272.211 & 38.156 & 39.723 & 163.038 & 174.618 \\
\hline \multicolumn{11}{|c|}{$\begin{array}{l}\text { Organic } N \text { management in } \\
I^{s t} \text { kharif crop }(N)\end{array}$} \\
\hline $\begin{array}{l}\mathrm{N}_{1}-75 \% \mathrm{~N} \text { as } \\
\text { vermicompost } \\
\end{array}$ & 0.580 & 0.719 & 5.381 & 5.781 & 260.743 & 267.568 & 35.275 & 38.301 & 158.695 & 169.152 \\
\hline $\begin{array}{l}\mathrm{N}_{2}-100 \% \mathrm{~N} \text { as } \\
\text { vermicompost } \\
\end{array}$ & 0.595 & 0.733 & 5.429 & 5.821 & 264.081 & 270.076 & 36.585 & 39.522 & 162.202 & 171.320 \\
\hline $\operatorname{SEm}( \pm)$ & 0.004 & 0.003 & 0.013 & 0.013 & 0.908 & 0.789 & 0.295 & 0.253 & 1.514 & 1.008 \\
\hline $\mathrm{CD}(\mathrm{P}=0.05)$ & 0.013 & 0.007 & 0.039 & 0.039 & 1.944 & 1.689 & 0.630 & 0.541 & 3.240 & 2.157 \\
\hline \multicolumn{11}{|c|}{$\begin{array}{l}\text { Organic P management in } \\
2^{\text {nd }} \text { kharif crop }(P)\end{array}$} \\
\hline $\begin{array}{l}P_{1-75}=P \text { as } \\
\text { vermicompost }\end{array}$ & 0.576 & 0.714 & 5.352 & 5.768 & 259.221 & 266.450 & 34.533 & 37.783 & 157.473 & 167.599 \\
\hline $\begin{array}{l}P_{2^{-}} 100 \% \mathrm{P} \text { as } \\
\text { vermicompost } \\
\end{array}$ & 0.597 & 0.738 & 5.458 & 5.833 & 265.604 & 271.194 & 37.327 & 40.040 & 163.424 & 172.873 \\
\hline $\operatorname{SEm}( \pm)$ & 0.004 & 0.003 & 0.012 & 0.013 & 1.580 & 0.951 & 0.282 & 0.240 & 1.320 & 0.644 \\
\hline $\mathrm{CD}(\mathrm{P}=0.05)$ & 0.014 & 0.006 & 0.036 & 0.039 & 3.381 & 2.035 & 0.597 & 0.514 & 2.799 & 1.378 \\
\hline Interactions & NS & NS & NS & NS & NS & NS & NS & NS & NS & NS \\
\hline \multirow[t]{2}{*}{$\mathrm{CV}(\%)$} & 3.582 & 1.489 & 1.177 & 1.095 & 1.199 & 1.017 & 2.840 & 2.250 & 3.269 & 2.051 \\
\hline & 3.749 & 1.378 & 1.075 & 1.098 & 2.085 & 1.226 & 2.715 & 2.140 & 2.850 & 1.310 \\
\hline
\end{tabular}




\section{Effect on yield}

Significantly higher yield performance of greengram under maize-greengram cropping system $\left(C_{2}\right)$ could be due to the fact that maize being a $\mathrm{C} 4$ plant, comparatively lower weed growth was observed (visual) this smothering effect on weed growth may have reduced soil nutrient uptake by weeds thereby resulting in residual effect of applied nutrients for succeeding kharif greengram crop. It may also be noted that subsequent phosphorous management in second kharif crop greengram might have resulted in positive additive reaction with residual effect of first crop. whereas, under rice-greengram system it was also visually observed that owing to smaller stature of rice plant weed growth was comparatively higher which may have led to increased removal of applied nutrients resulting in sub optimal availability of residual nutrient and yield of succeeding greengram crop. Higher yield, yield attributes of greengram and rice and maize equivalent yields recorded by the systems under the treatments $\mathrm{N}_{2}$ and $\mathrm{P}_{2}$ may be attributed to the fact that optimum doses of nitrogen under $\mathrm{N}_{2}$ applied through vermicompost to the preceding kharif crops (rice and maize) may have resulted in residual carry-over of nutrients to the succeeding kharif greengram this, coupled with optimum phosphorous application $\left(\mathrm{P}_{2}\right)$ might have boosted the yield performance of greengram and the equivalent yields of the systems. The significant residual effect of vermicompost application on the succeeding greengram and other crops on yield and yield attributes were also reported by Faujdar and Sharma (2013), Dey and Paul, (2013), Pate et al., (2014) and Alagappan and Venkitaswamy (2016). The efficacy of vermicompost application on increasing the yield of greengram was also reported by Rajkhowa et al., (2002), Bhatt et al., (2012) and Sushil et al., (2015), which confirms to the findings of the present investigation.

\section{Effect on soil health}

The findings on the effect of treatments on the soil nutrient status suggests that there was a significant effect on all the crops in sequence with regard to organic $\mathrm{C}, \mathrm{pH}$ and available $\mathrm{N}$, $\mathrm{P}_{2} \mathrm{O}_{5}$ and $\mathrm{K}_{2} \mathrm{O}$ content in the soil. Significantly higher build-up of soil organic $\mathrm{C}, \mathrm{pH}$ and available $\mathrm{N}, \mathrm{P}_{2} \mathrm{O}_{5}$ and $\mathrm{K}_{2} \mathrm{O}$ under maize-greengram sequence as observed under both first and second kharif crop may be due to the fact that maize crop could significantly supress the weed population thereby resulting in lesser uptake of soil nutrients by the weeds as compared to rice-greengram sequence. The application of $100 \% \mathrm{~N}$ and $\mathrm{P}$ through vermicompost showed significant influence which resulted in higher soil organic $\mathrm{C}, \mathrm{pH}$ and available $\mathrm{N}, \mathrm{P}_{2} \mathrm{O}_{5}$ and $\mathrm{K}_{2} \mathrm{O}$ content as compared with the application of $75 \% \mathrm{~N}$ and $\mathrm{P}$ through vermicompost which may be attributed to availability of higher soil nutrients with application of $100 \% \mathrm{~N}$ and $\mathrm{P}$ as compared to $75 \% \mathrm{~N}$ and $\mathrm{P}$ in both the crop sequence. It was observed that there was a steady built up of organic $\mathrm{C}, \mathrm{pH}$ and available $\mathrm{N}, \mathrm{P}_{2} \mathrm{O}_{5}$ and $\mathrm{K}_{2} \mathrm{O}$ content both before sowing and after harvest of greengram crop during both the years. This may be due to combined effect of vermicompost in the first kharif crop (rice and maize) followed by application of vermicompost in the second kharif crop (greengram) which may have increased their availability and subsequent build. These findings of the present investigation corresponds to the findings conducted by Parthasarathi et al., (2003), Rajkhowa et al., (2002), Singh et al., (2005), Jayaprakash et al., (2004), Parthasarathi et al., (2008), Ramesh et al., (2010), Porpavai et al., (2011), Vidyavathi et al., (2011), Tharmaraj et al., (2011), Kachroo et al., (2014), Choudhary and Kumar (2013), where they reported that application of vermicompost increased the organic $\mathrm{C}, \mathrm{pH}, \mathrm{N}, \mathrm{P}_{2} \mathrm{O}_{5}$ and $\mathrm{K}_{2} \mathrm{O}$ content in the soil. They also reported that with the 
increase in the level of vermicompost, the soil organic $\mathrm{C}, \mathrm{pH}, \mathrm{N}, \mathrm{P}_{2} \mathrm{O}_{5}$ and $\mathrm{K}_{2} \mathrm{O}$ content tends to increase.

From the findings of the present investigation it may be concluded that organic management of rainfed cropping system encourages buildup of soil organic $\mathrm{C}, \mathrm{pH}$ and available $\mathrm{N}$, $\mathrm{P}_{2} \mathrm{O}_{5}$ and $\mathrm{K}_{2} \mathrm{O}$ in soilthereby maintaining and improving the soil health. Maize-greengram cropping system with organic management of nutrients viz., $100 \% \quad \mathrm{~N}$ and $\mathrm{P}$ through vermicompost, is a better alternative than rice-greengram cropping system under rainfed condition of north-east hill region for maximizing productivity and profitability of the system.

\section{References}

Alagappan and Venkitaswamy, R. 2016. Impact of different sources of organic manures in comparison with TRRI practice, RDF and INM on growth, yield and soil enzymatic activities of rice - greengram cropping system under site - specific organic farming situation. American-Eurasian Journal of Sustainable Agriculture 10(2): 1-8.

Bhatt, P. K., Patel, B. T., Ravel, C. H and Vyas, K. G. 2012. Effect of vermicompost and phosphorus levels with PSB on growth and yield of summer greengram (Vigna radiata $\mathrm{L}$.) under north Gujarat conditions. Green Farming 3(6): 666-669.

Chaudhary, V. K and Kumar, P. S. 2013. Maize production, economics and soil productivity under different organic source of nutrients in Eastern Himalayan Region, India. International Journal of Plant Production 7(2): 167186.

Das, A., Patel, D. P., Munda, G. C., Ghosh, P. K., Ngachan, S. V., Choudhury, B.U., Ramkrushna, G. I., Saha, R., Rajkhowa,
D. J., Pamwar, A. S., Kumar, R., Kumar, M and Juri, B. 2011. Conservation agriculture in rice and maize based cropping systems: Principles and practices for $\mathrm{NEH}$ Region. Research Bulletin No. 75.

Dey, J. K. and Paul, S. R. 2013. Effect of insitu greengram (Phaseolus radiatus) residue and nitrogen levels on toria (Brassica rapa) in rice (Oryza sativa) greengram-toria sequence in Ultic Haplustalf. The Indian Journal of Agricultural Sciences 46(3): 67-74.

Faujdar, R. S. and Sharma, M. 2013. Effect of FYM, biofertilizers and zinc on yield of maize and their residual effect on wheat. Journal of Soils and Crops 23(1): 41-52.

Jayaprakash, D. C., Sawant, P. S. and Singh, R. S. 2004. Effect of vermicompost on growth and yield of maize as well as nutrient uptake. Indian Journal of Agronomy 23(1):121-123.

Kachroo, D., Thakur, N. P., Kour, M., Kumar, P., Sharma, R. and Khajuria, V. 2014. Diversification of rice (Oryza sativa L.) - based cropping system for enhancing productivity and employment. Indian Journal of Agronomy 59(1): 21-25.

Parthasarathi, K., Balamurugan, $\mathrm{M}$ and Ranganathan, L. S. 2008. Influence of vermicompost on the physico-chemical and biological properties in different types of soil along with yield and quality of the pulse crop-Blackgram. Iranian Journal of Environmental Health Science \&Engineering 5(1): 51-58

Parthasarathi, K., Balamurugan, M. and Ranganathan, L. S. 2003. Influence of vermicompost on the physico-chemical and biological properties in different types of soil along with yield and quality of pulse crop blackgram. Journal of sustainable Agriculture 23(1): 51-58. 
Patel, H. K., Sadhu, A. C., Lakum, Y. C. and Suthar, J. V. 2014. Response of integrated nutrient management on wheat (Triticum aestivum L.) and its residual effect on succeeding crop. International Journal on Agriculture Sciences and Veterinary Medicines 2(4): 47-52.

Porpavai1, S., Devasenapathy, P., Siddeswaran, K. and Jayaraj, T. 2011. Impact of various rice based cropping systems on soil fertility. Journal of Cereals and Oilseeds 2(3): 43-46.

Rajkhowa, D. J., Saikia, M. and Rajkhowa, K. M. 2002. Effect of vermicompost and levels of fertilizer on greengram. Legume Research 26(1): 63-65.

Ramesh, P., Panwar, N. R., Singh, A. B., Ramana, S., Sushil, K. Y., Rahul, S. and Subha, R. 2010. Status of organic farming in India.Current Science. 8(9): 1190-1194.

Singh, G., Jalota, S. K. and Sidhu, B. S. 2005. Soil physical and hydraulic properties in a rice-wheat cropping system in India: effect of rice straw management. Soil Use and Management 21: 17-21.

Sushil, V., Lal, E. P. and Rao, K. P. 2015. Studies on integrated nutrient management on seed yield and quality of greengram (Vigna Radiate L.). International Journal of Recent Research in Life Sciences 2(2): 42-45.

Tharmaraj, K., Ganesh, P., Kolanjinathan, K., Suresh Kumar, R. and Anandan, A. 2011. Influence of vermicompost and vermiwash on physico chemical properties of rice cultivated soil. Current Botany 2(3): 18-21.

Vidyavathi, G., Dasog, S., Babalad, H. B., Hebsur, N. S., Gali, S. K., Patil, S. G. and Alagawadi, A. R. 2011. Influence of nutrient management practices on crop response and economics in different cropping systems in a vertisol. Karnataka Journal of Agricultural Science 24(4): 455-460.

\section{How to cite this article:}

Khumdemo Ezung, N., J.K. Choudhary, K. Kurmi, Noyingthung Kikon and Moasunep. 2018. Efficacy of Organically Managed Cropping System in Improvement of Soil Health in Ne Hill Region. Int.J.Curr.Microbiol.App.Sci. 7(09): 157-169. doi: https://doi.org/10.20546/ijcmas.2018.709.020 